ing, that are not superconductors on their own, but rather exhibit superconductivity at the interface between them. The layer identified as essential to the superconductivity by the zinc-substitution experiment represents the second copper-oxide layer away from the interface.

The scientists found that the presence of zinc had no effect on the transition temperature at which superconductivity sets in, $32 \mathrm{~K}\left(-241^{\circ} \mathrm{C}\right)$, except when placed in that particular layer. In the latter case, the scientists observed a dramatic drop in the transition temperature to $18 \mathrm{~K}\left(-255^{\circ} \mathrm{C}\right)$. The reduction in transition temperature provides a clear indication that that particular layer is the "hot" one responsible for the relatively high temperature at which superconductivity normally sets in for this material, according to the researchers.

"We now have a clean experimental proof that high-temperature superconductivity can exist, undiminished, in a single copper-oxide layer," Božović said. "This piece of information gives important input to our theoretical understanding of this phenomenon."

Božović said that, in the material he studied, the electrons required for superconductivity actually come from the metallic material below the interface. They leak into the insulating material above the interface and achieve the critical level in that second copper-oxide layer.

But in principle, he said, there are other ways to achieve the same concentration of electrons in that single layer, for example, by doping achieved by applying electric fields. That would result in high-temperature superconductivity in a single copperoxide layer measuring just $0.66 \mathrm{~nm}$.

From a practical viewpoint, this discovery opens a path toward the fabrication of electronic devices with modulated, or tunable, superconducting properties which can be controlled by electric or magnetic fields, said the researchers.

"Electronic devices already consume a large fraction of our electricity usageand this is growing fast," Bozovic said. "Clearly, we will need less-power hungry electronics in the future."

Superconductors, which operate without energy loss-particularly those that operate at warmer, more-practical temperatures-may be one way to go.

According to the researchers, their layer-by-layer synthesis method and ability to strategically alter individual layers' composition might also be used to explore and possibly control other electronic phenomena and properties that emerge at the interfaces between layered materials.

\section{Hierarchical Topographies Created by Controlled Evaporation of a Block Copolymer Solution}

Hierarchically ordered materials tend to have unique physical properties. For instance, the lotus leaf has both microand nanostructures which contribute to its superhydrophobicity. To produce materials with useful properties such as superhydrophobicity, simple methods for making hierarchical materials must be established. Block copolymers provide a way of creating nano-patterned surfaces because of their ability to self-assemble into their constituent blocks. Zhiqun Lin, Suck Won Hong, and Jun Wang at Iowa State University have shown that controlled evaporation of a diblock copolymer solution creates a serpentine-like microstructure, which can further be processed to have nanostructures.

As described in the October 19 issue of Angewandte Chemie International Edition (DOI: 10.1002/anie.200903552; p. 8356), Lin and co-workers prepared a solution of polystyrene-block-poly(methylmetha- crylate) (PS- $b$-PMMA) in toluene at a concentration of $0.13 \mathrm{mg} / \mathrm{mL}$. The molecular weight of each block was $45.9 \mathrm{~kg} / \mathrm{mol}$ and $138 \mathrm{~kg} / \mathrm{mol}$ for PS and PMMA, respectively. This solution was held between a fused silica lens (1 cm diameter) and a silicon wafer by capillary forces. As the solution evaporated toward the center of the lens, concentric rings of the block copolymer were deposited on the silicon wafer surface. These rings had an undulating or "serpentine" morphology on the micron scale. The spacing between the characteristic features was approximately $20 \mu \mathrm{m}$ and the height was approximately $100 \mathrm{~nm}$. To further change the morphology, the researchers aged the films by exposing them to acetone vapor for up to 12 hours. After exposing the films to acetone vapor, the microtopography changed from "serpentine-like" to "mesh-like" —with distinct holes forming the "mesh-like" structure. At the nanoscale, atomic force microscopy and transmission electron microscopy imaging showed that the acetone vapor caused the polystyrene blocks to phase- segregate into hexagonally packed, cylindrical nanodomains $\sim 39 \mathrm{~nm}$ in diameter. The surface chemistry was analyzed by x-ray photoelectron spectroscopy (XPS) and showed that aging the films with acetone vapor increases the carbonyl $(\mathrm{O}-\mathrm{C}=\mathrm{O})$ signal at $289.1 \mathrm{eV}$ relative to the hydrocarbon (C-C or C-H) signal at $285.0 \mathrm{eV}$.

The researchers attributed the "serpentine-like" microstructure to fingering instabilities which arise during progressive "stick-slip" motion of the three phase contact line as the solvent evaporates. The researchers propose that polystyrene initially forms at the top of the film due to its lower surface tension. XPS results demonstrated that the PMMA matrix reorients toward the surface after exposure to acetone vapor. This caused the polystyrene to form cylindrical nanodomains oriented perpendicular to the surface. Lin and colleagues propose that controlled self-assembly methods such as these allow for simple fabrication of hierarchical structures without using lithography.

SCOTT COOPER

\section{How to Choose In-plane Ferroelectric Polarization States in Rhombohedral $\mathrm{BiFeO}_{3}$}

An international team of researchers have devised a way to reliably manipulate the ferroelastic polarization states of rhombohedral multiferroic materials that allows coupling to the strain and magnetic properties of these materials.

"The control of polarization switching to create different domain patterns with predefined in-plane domain orientations is key to applications which are based on the physical properties of domain walls or on the coupling of ferroic order parameters," said N. Balke of Oak Ridge National Laboratory in explaining the potential impact of their discovery.

As reported in the October 11 on-line issue of Nature Nanotechnology (DOI: 10.1038/NNANO.2009.293), the researchers used the electric field from a moving piezoresponse force microscope (PFM) tip to deterministically generate domain states in $\mathrm{BiFeO}_{3}(\mathrm{BFO})$, including long-sought closure domains. Closure domains are ferroic domains of position and orientation such that flux lines from larger, adjacent domains close upon themselves (see, e.g, www.answers.com/topic/closuredomain). The tip motion that broke the rotational symmetry of the electric field was a crucial element that allowed the 
researchers to achieve this advancement compared to previous studies.

Few materials allow the manipulation of the strain and magnetic states of a multiferroic through direct application of an electric field, because the stable polarization states in most materials are antiparallel and couple similarly to the other order parameters. This is not so for rhombohedral structures, which can switch to one of four energetically equivalent up- or downpolarized states. M. Huijben, Y.H. Chu, and R. Ramesh of the University of California, Berkeley synthesized samples of rhombohedral (100) BFO to take advan- tage of this. The BFO was deposited atop leads composed of $\mathrm{SrRuO}_{3}(\mathrm{SRO})$ all on a $\mathrm{TiO}_{2}$-terminated (001) $\mathrm{SrTiO}_{3}$ (STO) substrate to promote growth of $\mathrm{BFO}$.

The phase field modeling of S. Choudhury and L.Q. Chen of Pennsylvania State University showed that all four polarization states of $\mathrm{BFO}$ can be accessed by the same switching field due to rotationally invariant symmetries in the material and the electric field using phase-field simulations. How then to manipulate the ferroelectric domains?

Balke and her co-workers at Oak Ridge, S. Jesse, A.P. Baddorf, and S.V. Kalinin, found that they could break the symmetry by applying a voltage to a moving PFM tip. They showed that they could repetitively produce domain patterns this way, including a line of closure domains.

"This work shows that it is possible to control the switching process to utilize all new possible functionalities," said Balke, "whether these are local strain sensors, multi-level resistance devices, or information storage devices that write electrically and read magnetically."

JIM RANTSCHLER

\section{News of MRS Members/Materials Researchers}

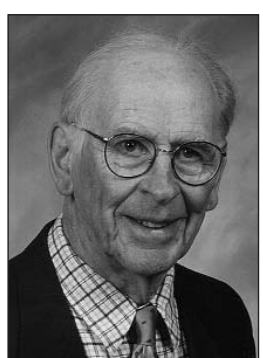

W. Conyers Herring
William Conyers Herring passed away at his home in Palo Alto, California, on July 23, 2009 at the age of 94 . For over five decades he made insightful contributions in both materials science and solid-state physicsa unique example of the value of interdisciplinary research. His theories of capillary phenomena, diffusionlimited plasticity in metals, mechanical behavior of small particles, sintering, and thermionic behavior were recognized in 1980 when he received the Materials Research Society Von Hippel Award with the citation, "Demonstration that whiskers of high crystalline perfection would exhibit extraordinary mechanical properties. He is also held in esteem for his theoretical contributions to the understanding of surfaces and surface tension."

In the same year he received the James Murray Luck Award for Excellence in Scientific Reviewing by the National Academy of Sciences in recognition of his scholarly, comprehensive reviews of thermionic, magnetic, and transport phenomena that are widely cited today.

Earlier in 1959 he was awarded the Oliver E. Buckley Award of the American Physical Society for his major contributions to electronic band theory and its applications.

When Conyers started school at five years old in the small town of Parsons, Kansas he had already taught himself how to read so well that he was placed in the fifth grade. His father's death when he was 13 left the family impoverished, but the following year he was able to enroll in the University of Kansas with a four-year scholarship. He graduated with a major in astronomy and then switched his focus to solid-state physics when he went to Princeton University, where he particularly enjoyed being able to study independently with few required courses. There, a small group including Eugene Wigner and his graduate students John Bardeen, Frederick Seitz, and Conyers created the modern band theory of solids. Conyers received his degree in 1937 and spent the following two years as a National Research Council fellow at the Massachusetts Institute of Technology. There he introduced the orthogonalized plane wave (OPW) method, the first workable scheme for calculating electronic energy bands in solids, and with A.G. Hill used it to calculate the band structure of beryllium.

Conyers served in the Division of War Research at Columbia University during the second World War. In 1946, after a short time in the Applied Mathematics Department at the University of Texas, he took a position as a member of the technical staff at the Bell Telephone Laboratories, Murray Hill, New Jersey. In that same year he married Louise Preusch who had just graduated from Barnard in math and physics. They made their home in nearby Summit, where their four children Lois, Alan, Brian, and Gordon were born and raised.

Conyers created the theoretical physics department from the strong group already existing at Bell that soon was recognized to be preeminent and a mecca for visitors coming from around the world. Conyers and Louise were gracious hosts and made many friends. He kept himself and others well informed of the rapid advances being made by reading the latest journals as soon as they arrived at the extensive Bell library. He organized references he considered significant by subjects on three-by-five cards containing multi-references with terse comments all written in long hand. In order to keep current with advances in Russia during the cold war (not wanting to wait for problematic translations) Conyers simply learned to read Russian. He regularly carried his card file in a famous black suitcase, willingly serving the community as a one-man Google-actually a more useful one, because he had already filtered out the extraneous. He was not only known as a fountain of knowledge, but also for his helpful way of sharing, treating everybody with respect and quietly providing his own keen insights. Wigner once remarked [to me] that whenever there was something he wanted to know (in solid state) the first thing he did was to go to Conyers. His review of exchange among itinerant electrons that started as a chapter and turned into a book on magnetism was one of the first recognitions of the role of collective excitations in metals. John H. Van Vleck in reviewing the book (Physics Today, April 1967) said that "the preprint at Harvard was so bulky it was usually called the telephone book because of its origin at Murray Hill and its accuracy, attention to detail and usefulness, but it has one detail that the telephone book lacks - the quality of being critical in the best sense of the word."

Conyers moved to Stanford University as Professor of Applied Physics in 1978. $\mathrm{He}$ and Louise became active members in the campus community, and he continued productive research activities. Conyers had many outside interests. He was an avid tennis player and a wit who could produce a clever limerick spontaneously. He was a devout Christian who believed that theology underlies science because "science is ultimately based on leaps of intuition and aesthetic perceptions." Those who knew him cherish memories of this remarkable man.

THEODORE H. GEBALLE 